\title{
On the periodic solution of a class of stochastic nonlinear system with delays
}

\author{
Bo Du ${ }^{\mathrm{a}, *}$, Haiyan Wang ${ }^{\mathrm{b}}$, Maoxing Liu ${ }^{\mathrm{c}}$, Xiwang Cheng ${ }^{\mathrm{a}}$ \\ ${ }^{a}$ Department of Mathematics, Huaiyin Normal University, Huaian Jiangsu, 223300, P. R. China. \\ ${ }^{b}$ School of Mathematical and Natural Sciences, Arizona State University, Arizona, USA. \\ ${ }^{c}$ Department of Mathematics, North University of China, Taiyuan 030051, P. R. China.
}

Communicated by K. Q. Lan

\begin{abstract}
This paper is devoted to investigating a class of stochastic nonlinear system with periodic coefficients. Some criteria on existence and uniqueness of periodic solution are established for the stochastic nonlinear system. Finally, a numerical example is given to show the effectiveness and merits of the present results.
\end{abstract}

Keywords: Periodic solution, stochastic, Itô's formula, existence.

2010 MSC: MSC 34K50.

(C)2018 All rights reserved.

\section{Introduction}

During the past years, the theory and applications of stochastic differential equations have been developed very quickly, see e.g. [3,11-13]. However, few results have been obtained in the direction of the periodically stochastic differential equations. Till now, we only find that very few results for periodic solution of stochastic differential equations have been published in $[5,6,19]$. Recently, some results have been established for the periodically stochastic differential equations. In [4] and [1], the authors studied the existence of periodic solution of differential equations with random right sides. $\mathrm{Xu}$ et al. [17] considered the following periodic stochastic functional differential equation (SFDE)

$$
\left\{\begin{array}{l}
\mathrm{d} x(\mathrm{t})=\mathrm{B}\left(\mathrm{t}, \mathrm{x}_{\mathrm{t}}\right) \mathrm{dt}+\sigma\left(\mathrm{t}, x_{\mathrm{t}}\right) \mathrm{dW}(\mathrm{t}), \mathrm{t} \geqslant \mathrm{t}_{0} \geqslant 0 \\
x_{\mathrm{t}_{0}}\left(\mathrm{t}_{0}+\mathrm{s}\right)=\phi(s) \in C, s \in[0, \tau]
\end{array}\right.
$$

\footnotetext{
*Corresponding author

Email addresses: dubo7307@163.com (Bo Du), Haiyan.Wang@asu.edu (Haiyan Wang), liumaoxing@126. com (Maoxing Liu), chengxiwang68@163.com (Xiwang Cheng)
}

doi: $10.22436 /$ jnsa.011.02.08

Received: 2017-11-17 Revised: 2017-12-03 Accepted: 2017-12-22 
on the probability space $\left(\Omega, \mathcal{F},\left\{\mathcal{F}_{t}\right\}_{t} \geqslant 0, P\right)$. By using the properties of periodic Markov processes, they obtained some sufficient conditions for the existence of periodic solution of system (1.1). After that, when the delay is unbounded in system (1.1), $\mathrm{Li}$ and $\mathrm{Xu}$ [9] also obtained the existence of periodic solution of system (1.1). In [19], Zhang and Gopalsamy studied two $n$ species stochastic population models with periodic coefficients. Based on Lemma 2.3 (see e.g. [2]), some existence results of periodic solution are obtained for the above system, but the conditions of Lemma 2.3 are very sharp. In [24], a class of impulsive stochastic Nicholson's blowflies model is investigated by applying Cauchy matrix. Under proper conditions, the existence and exponential stability of square-mean almost periodic solutions for the model with multiple nonlinear harvesting terms and delays.

For practical applications, we note that when designing the neural networks or in the implementation of neural systems, stochastic perturbations are almost inevitable [10]. Hence, stochastic modeling is a vital issue. Therefore, it is necessary to investigate effects of stochastic perturbations on the stability of neural networks. Stability analysis of various stochastic neural networks with time delays has become an attractive topic of research, see e.g. [14, 16, 22, 23]. In [8], the stability and stabilization were studied for a class of stochastic systems with impulsive effects. In [15], the pth moment $(p \geqslant 2)$ and the almost-sure stability of stochastic Cohen-Grossberg neural networks with mixed time delays and nonlinear impulsive effects were investigated through the Razumikhin type technique. Zhang et al. [21] studied a class of stochastic neural networks with local impulsive effects. For deterministic neural network, see e.g. [18, 20].

One of our main objectives is to study a kind of stochastic nonlinear system and obtain the properties of periodic solution for the above system. Firstly, an effective existence theorem for stochastic periodic process is established. Then, some sufficient conditions for the uniqueness of periodic solution of nonlinear system are given. To overcome the difficulties created by the special features possessed by the periodic stochastic equations with delays, as one will see, several novel analysis methods are introduced. These existence theorems are rather general and therefore have great power in applications.

The distinctive contributions of this paper are outlined as follows: (1) few results are obtained for the existence and uniqueness of the periodic solutions of stochastic nonlinear system with activation functions, novel analysis technique is developed since the conventional analysis tool no longer applies; (2) we develop the methods in [17] and [9], our method for the proof of existence and uniqueness of periodic solutions can more easily be understood; (3) the existence theorems of stochastic periodic solutions in this paper are rather general and therefore have great power in applications.

The following sections are organized as follows. In Section 2, some useful Lemmas and Definitions are given. In Section 3, sufficient conditions are established for existence and uniqueness of periodic solutions of systems (3.1) and (3.9), respectively. In Section 4, an example is given to show the feasibility of our results.

\section{Preliminaries}

In [7], Kolmanovskii and Myshkis have considered the existence of periodic solutions of the SFDEs of Itô. The method of the shift and averaging of the initial distribution generating a solution with certain properties was used. Particularly, they considered SRDE (stochastic retard differential equation) of the form

$$
\left\{\begin{array}{l}
d x(t)=a\left(t, x_{t}\right) d t+b\left(t, x_{t}\right) d \xi(t), t \geqslant 0, \\
x_{t}(\theta):=x(t+\theta),-h \leqslant \theta \leqslant 0, x(t) \in \mathbb{R}^{n}, \xi(t) \in \mathbb{R}^{l},
\end{array}\right.
$$

with the initial condition

$$
x_{\mathrm{t}}(\theta):=\phi(\theta),-\mathrm{h} \leqslant \theta \leqslant 0 .
$$

Here $\xi(t)$ is the standard Wiener process, the continuous functionals $a(t, \phi)$ and $b(t, \phi)$ are defined on $[0, \infty) \times C[-h, 0]$ and are T-periodic with respect to the first argument. The solution of system (2.1) for $t \geqslant 0$ is determined by initial condition (2.2). Now we give some basic results for the periodic solutions of the SFDEs of Itô. 
Definition 2.1 ([7]). T-periodic solution of (2.1) is a T-periodic stochastic process periodically connected with $\xi(t)$ and satisfying (2.1) with probability 1 . The form means that all finite-dimensional distributions of the process $(x(t),-\xi(\tau)+\xi(s)), \tau<s$, are invariant with respect to all shifts of the arguments by the quantity $k T$, where $k$ is an arbitrary integer, i.e., that for any positive integers $N, m$ and any $t_{1}, \ldots, t_{N}, \tau_{1}, \ldots, \tau_{m}, s_{1}, \ldots, s_{m}$ such that $\tau_{i}<s_{i}, i=1, \ldots, m$, the distribution of the probabilities of the random variable $\left(x\left(t_{1}\right), \ldots, x\left(t_{N}\right), \xi\left(s_{1}\right)-\xi\left(\tau_{1}\right), \ldots, \xi\left(s_{m}\right)-\xi\left(\tau_{m}\right)\right)$ coincides with the distribution of the probabilities of variable $\left(x\left(t_{1}+k T\right), \ldots, x\left(t_{N}+k T\right), \xi\left(s_{1}+k T\right)-\xi\left(\tau_{1}+k T\right), \ldots, \xi\left(s_{m}+k T\right)-\xi\left(\tau_{m}+k T\right)\right)$.

Theorem 2.2 ([7]). Assume that the the coefficients of system (2.1) satisfy the assumptions

$$
|\mathrm{a}(\mathrm{t}, \phi)|^{2} \leqslant \mathrm{q}_{1}+\int_{-\mathrm{h}}^{0}|\phi(\mathrm{s})|^{2} \mathrm{dk}_{1}(\mathrm{~s}),|\mathrm{b}(\mathrm{t}, \phi)|^{2} \leqslant \mathrm{q}_{2}+\int_{-\mathrm{h}}^{0}|\phi(\mathrm{s})|^{2} \mathrm{dk}_{2}(\mathrm{~s}),
$$

where $\mathrm{q}_{1}$ and $\mathrm{q}_{2}$ are some positive constants, and $\mathrm{k}_{1}(\mathrm{~s})$ and $\mathrm{k}_{2}(\mathrm{~s})$ are scalar non-decreasing functions on $[-\mathrm{h}, 0]$. Let, further on, a solution $x(t, \phi)$ of (2.1) and (2.2) exists such that for $s_{1}, s_{2} \in[-h, 0]$,

$$
\mathbb{E}\left|x\left(s_{1}\right)-x\left(s_{2}\right)\right|^{\varepsilon_{1}} \leqslant C\left|s_{1}-s_{2}\right|^{1+\varepsilon_{2}}
$$

where $\mathbb{E}$ is mathematical expectation, $\varepsilon_{1}, \varepsilon_{2}$ and $\mathrm{C}$ are positive constants, and for $\mathrm{t} \geqslant 0$ the second moment is bounded:

$$
\mathbb{E}|x(t, \phi)|^{2} \leqslant C<\infty .
$$

Then there exists a T-periodic solution of system (2.1).

Lemma 2.3 ([7]). Let the continuous scalar functions $z(t)$ and $f(t), t \geqslant 0$ satisfy for some constant $C$ and for any $\mathrm{t}_{1}, \mathrm{t}_{2}: \mathrm{t}_{2} \geqslant \mathrm{t}_{1} \geqslant 0$ the inequality

$$
z\left(t_{2}\right)-z\left(t_{1}\right) \leqslant-C \int_{t_{1}}^{t_{2}} z(s) d s+\int_{t_{1}}^{t_{2}} f(s) d s .
$$

Then

$$
z(\mathrm{t}) \leqslant z(0) e^{-\mathrm{Ct}}+\int_{0}^{t} e^{-\mathrm{C}(\mathrm{t}-\mathrm{s})} \mathrm{f}(\mathrm{s}) \mathrm{d} \mathrm{s} .
$$

Lemma 2.4 ([7]). Let the continuous non-negative function $x(t)$ satisfies for some constants $C_{i}$ the inequality

$$
x(t) \leqslant C_{1}+C_{2} \int_{0}^{t} e^{-C_{3}(t-s)} x(s) d s .
$$

Then, if $\mathrm{C}_{3}>\mathrm{C}_{2}$, then $x(\mathrm{t}) \leqslant \mathrm{C}_{3} \mathrm{C}_{1}\left(\mathrm{C}_{3}-\mathrm{C}_{2}\right)^{-1}$.

Remark 2.5. Theorem 2.2 shows that the boundedness of the moments of some solution implies the existence of a periodic solution. In relation to Theorem 2.2 we shall note that for determinate equation of the form (2.1) for $b=0$ the existence of a bounded solution does not imply the existence of a periodic solution.

\section{Existence and stability of periodic solutions}

In this section, we consider a stochastic nonlinear system

$$
\left\{\begin{aligned}
\mathrm{d} x_{i}(t)= & {\left[-a_{i}(t) x_{i}(t)+\sum_{j=1}^{n} a_{i j}(t) f_{i}\left(x_{i}(t)\right)+\sum_{j=1}^{n} d_{i j}(t) g_{i}\left(x_{i}(t-\tau)\right)\right] d t } \\
& +\sigma_{i}\left(t, x_{i}(t), x_{i}(t-\tau)\right) d \xi_{i}(t), \\
x_{i t}(\theta)= & \phi_{i}(\theta), \quad \theta \in[-h, 0], i=1,2, \ldots, n,
\end{aligned}\right.
$$

where $t \geqslant 0, \phi_{i}(\theta) \in C([-h, 0], \mathbb{R}), 0<\tau \leqslant h, x_{i}(t)$ is the state of the system, $f_{i}\left(x_{i}(t)\right)$ and $g_{i}\left(x_{i}(t-\tau)\right)$ are the activation functions, $a_{\mathfrak{i}}(t) \geqslant 0$ shows the rate of change for the state $x(t), \tau$ represents the transmission delay, and $\xi(t)=\left(\xi_{1}(t), \ldots, \xi_{n}(t)\right)^{\top}$ is the standard Wiener process. We assume that $a_{i}(t), a_{i j}(t)$ and $d_{i j}(t)$ are defined on $[0, \infty)$, are T-periodic and continuous functions. Assume that the following conditions hold. 
$\left(\mathrm{H}_{1}\right)$ For $i=1,2, \ldots, n$, there exist positive constants $q_{1 i}$ and $q_{2 i}$ such that

$$
\left|g_{i}\left(x_{i}(t-\tau)\right)\right|^{2} \leqslant q_{1 i}+\int_{-h}^{0}\left|\phi_{i}(s)\right|^{2} d k_{1 i}(s), \quad\left|\sigma_{i}\left(t, x_{i}(t), x_{i}(t-\tau)\right)\right|^{2} \leqslant q_{2 i}+\int_{-h}^{0}\left|\phi_{i}(s)\right|^{2} d k_{2 i}(s),
$$

where $k_{1 i}(s)$ and $k_{2 i}(s)$ are scalar non-decreasing bounded functions on $[-h, 0]$.

$\left(\mathrm{H}_{2}\right)$ For $i=1,2, \ldots, n, t \geqslant 0$, we have

$$
\sum_{j=1}^{n} a_{i j}(t) \geqslant 0, x_{i}(t) f_{i}\left(x_{i}(t)\right) \leqslant 0 .
$$

$\left(\mathrm{H}_{2}\right)^{*}$ For $i=1,2, \ldots, n, t \geqslant 0$, we have

$$
\sum_{j=1}^{n} a_{i j}(t) \leqslant 0, x_{i}(t) f_{i}\left(x_{i}(t)\right) \geqslant 0 .
$$

Theorem 3.1. Let the assumptions $\left(\mathrm{H}_{1}\right)$ and $\left(\mathrm{H}_{2}\right)$ hold. Furthermore, the following inequality holds:

$$
\gamma_{1 i}=\inf _{t \geqslant 0}\left[a_{i}(t)-\alpha_{01}^{1 / 2} \sum_{j=1}^{n}\left|d_{i j}(t)\right| / 2-\alpha_{01}^{1 / 2} / 2-\alpha_{02} / 2\right]>0,
$$

where $\mathrm{j}=1, \ldots, \mathrm{n}, \alpha_{0 j}=\int_{-\mathrm{h}}^{0} \mathrm{dk}_{\mathrm{ji}}(\mathrm{s}), \mathrm{j}=1,2,3$, and $\mathrm{k}_{1 \mathrm{i}}$ and $\mathrm{k}_{2 \mathrm{i}}$ are scalar non-decreasing functions bounded on $[-\mathrm{h}, 0]$. Then there exists a T-periodic solution of (3.1).

Proof. Let

$$
k_{3 i}=\alpha_{01}^{-1 / 2} \sum_{j=1}^{n} d_{i j}(s) k_{1 i}(s)+k_{2 i}(s), i=1,2, \ldots, n .
$$

For $i=1,2, \ldots, n$, consider the following functional on the trajectories of (3.1):

$$
V\left(t, x_{i t}\right)=x_{i}^{2}(t)+\int_{-h}^{0} d k_{3 i}(s) \int_{t+s}^{t} x_{i}^{2}(\tau) d \tau,
$$

where $x_{i t}(\theta)=x_{i}(t+\theta), \theta \in[-h, 0]$. From Itô's formula, $\left(\mathrm{H}_{2}\right),(3.1)$, and (3.2), we have

$$
\begin{aligned}
& d V\left(t, x_{i t}\right) \\
& =\left[\sigma_{i}^{2}\left(t, x_{i}(t), x_{i}(t-\tau)\right)+\left(-a_{i}(t) x_{i}(t)+\sum_{j=1}^{n} a_{i j}(t) f_{i}\left(x_{i}(t)\right)+\sum_{j=1}^{n} d_{i j}(t) g_{i}\left(x_{i}(t-\tau)\right)\right) 2 x_{i}(t)\right. \\
& \left.\quad+x_{i}^{2}(t)\left(\sqrt{\alpha_{01}}+\alpha_{02}\right)-\int_{-h}^{0} x_{i}^{2}(t+s) d k_{3 i}(s)\right] d t+2 x_{i}(t) \sigma_{i}\left(t, x_{i}(t), x_{i}(t-\tau)\right) d \xi_{i}(t) \\
& \leqslant\left[\sigma_{i}^{2}\left(t, x_{i}(t), x_{i}(t-\tau)\right)+2 \sum_{j=1}^{n} d_{i j}(t) x_{i}(t) g_{i}\left(x_{i}(t-\tau)\right)\right. \\
& \left.\quad+x_{i}^{2}(t)\left(\sqrt{\alpha_{01}}+\alpha_{02}-2 a_{i}(t)\right)-\int_{-h}^{0} x_{i}^{2}(t+s) d k_{3 i}(s)\right] d t+2 x_{i}(t) \sigma_{i}\left(t, x_{i}(t), x_{i}(t-\tau)\right) d \xi_{i}(t) .
\end{aligned}
$$

We shall transform the separate addends in (3.3). In view of $\left(\mathrm{H}_{1}\right)$, considering the term

$$
2 x_{i}(t) \sum_{j=1}^{n} d_{i j}(t) g_{i}\left(x_{i}(t-\tau)\right),
$$


we have

$$
\begin{aligned}
& 2\left|x_{i}(t) \sum_{j=1}^{n} d_{i j}(t) g_{i}\left(x_{i}(t-\tau)\right)\right| \\
& \quad \leqslant \alpha_{01}^{1 / 2} \sum_{j=1}^{n}\left|d_{i j}(t)\right| x_{i}^{2}(t)+\alpha_{01}^{-1 / 2} \sum_{j=1}^{n}\left|d_{i j}(t)\right| g_{i}^{2}\left(x_{i}(t-\tau)\right) \\
& \quad \leqslant \alpha_{01}^{1 / 2} \sum_{j=1}^{n}\left|d_{i j}(t)\right| x_{i}^{2}(t)+\alpha_{01}^{-1 / 2} \sum_{j=1}^{n}\left|d_{i j}(t)\right|\left(q_{1 i}+\int_{-h}^{0}\left|\phi_{i}(s)\right|^{2} d k_{1 i}(s)\right) \\
& \quad=\alpha_{01}^{1 / 2} \sum_{j=1}^{n}\left|d_{i j}(t)\right| x_{i}^{2}(t)+\alpha_{01}^{-1 / 2} \sum_{j=1}^{n}\left|d_{i j}(t)\right|\left(q_{1 i}+\int_{-h}^{0}\left|x_{i t}(s)\right|^{2} d k_{1 i}(s)\right) \\
& \quad=\alpha_{01}^{1 / 2} \sum_{j=1}^{n}\left|d_{i j}(t)\right| x_{i}^{2}(t)+\alpha_{01}^{-1 / 2} \sum_{j=1}^{n}\left|d_{i j}(t)\right|\left(q_{1 i}+\int_{-h}^{0}\left|x_{i}(t+s)\right|^{2} d k_{1 i}(s)\right) \\
& \leqslant \alpha_{01}^{1 / 2} \sum_{j=1}^{n}\left|d_{i j}(t)\right| x_{i}^{2}(t)+\alpha_{01}^{-1 / 2} \sum_{j=1}^{n}\left|d_{i j}(t)\right| q_{1 i}+\alpha_{01}^{-1 / 2} \sum_{j=1}^{n}\left|d_{i j}(t)\right| \int_{-h}^{0}\left|x_{i}(t+s)\right|^{2} d k_{1 i}(s)
\end{aligned}
$$

and

$$
\begin{aligned}
\sigma_{i}^{2}\left(t, x_{i}(t), x_{i}(t-\tau)\right) & \leqslant q_{2 i}+\int_{-h}^{0}\left|\phi_{i}(s)\right|^{2} d k_{2 i}(s) \\
& =q_{2 i}+\int_{-h}^{0}\left|x_{i t}(s)\right|^{2} d k_{2 i}(s) \leqslant q_{2 i}+\int_{-h}^{0}\left|x_{i}(t+s)\right|^{2} d k_{2 i}(s), i=1, \ldots, n,
\end{aligned}
$$

where $x_{i t}(s):=\phi_{i}(s),-h \leqslant s \leqslant 0$. In view of (3.3)-(3.5), we have

$$
d V\left(t, x_{i t}\right) \leqslant-2 \gamma_{1 i} x_{i}^{2}(t) d t+C_{1 i} d t+2 x_{i}(t) \sigma_{i}\left(t, x_{i}(t), x_{i}(t-\tau)\right) d \xi_{i}(t),
$$

where

$$
\begin{aligned}
& \gamma_{1 i}=\inf _{t \geqslant 0}\left[a_{i}(t)-\alpha_{01}^{1 / 2} \sum_{j=1}^{n}\left|d_{i j}(t)\right| / 2-\alpha_{01}^{1 / 2} / 2-\alpha_{02} / 2\right]>0, i=1, \ldots, n, \\
& C_{1 i}=q_{2 i}+q_{1 i} \alpha_{01}^{-1 / 2} \sup _{t \geqslant 0}\left\{\sum_{j=1}^{n}\left|d_{i j}(t)\right|\right\}, i=1, \ldots, n .
\end{aligned}
$$

For $t_{2} \geqslant t_{1} \geqslant 0$, integrating the both sides of (3.6) from $t_{1}$ to $t_{2}$, and taking the mathematical expectation, we have

$$
\mathbb{E} x_{i}^{2}\left(t_{2}\right)-\mathbb{E} x_{i}^{2}\left(t_{1}\right) \leqslant-\gamma_{2 i} \int_{t_{1}}^{t_{2}} \mathbb{E} x_{i}^{2}(t) d t+C_{1 i}\left(t_{2}-t_{1}\right)+\int_{t_{1}}^{t_{2}} d t \int_{-h}^{0} \mathbb{E} x_{i}^{2}(t+s) d k_{3 i}(s),
$$

where $\gamma_{2 i}=2 \gamma_{1 i}+\alpha_{03}$. From (3.7) and Lemma 2.3, we have

$$
\mathbb{E} x_{i}^{2}(t) \leqslant C_{2 i}+\int_{0}^{t} d \tau \int_{-h}^{0} \mathbb{E} x_{i}^{2}(\tau+s) z_{i}(t-\tau) d k_{3 i}(s),
$$

where $C_{2 i}=C_{1 i} / \gamma_{2 i}+\mathbb{E} x_{i}^{2}(0), \quad z_{\mathfrak{i}}(t-\tau)=e^{-\gamma_{2 i}(t-\tau)}$. Denote by $v_{\mathfrak{i}}$ a function such that $v_{\mathfrak{i}}(t)=0$ for $t<0$ and $v_{\mathfrak{i}}(t)=1$ for $t \geqslant 0$ and set

$$
\Gamma_{\mathfrak{i}}(\tau)=\sup _{0 \leqslant t \leqslant \tau} \mathbb{E} x_{i}^{2}(t), i=1, \ldots, n
$$


Note that

$$
\begin{aligned}
\int_{0}^{t} \mathrm{~d} \tau \int_{-h}^{0} \mathbb{E} x_{i}^{2}(\tau+s) z_{i}(t-\tau) d k_{3 i}(s)= & \int_{0}^{t} d \tau \int_{-h}^{0} \mathbb{E} \phi_{i}^{2}(\tau+s) z_{i}(t-\tau)\left[1-v_{i}(s+\tau)\right] d k_{3 i}(s) \\
& +\int_{0}^{t} d \tau \int_{-h}^{0} \mathbb{E} x_{i}^{2}(\tau+s) z_{i}(t-\tau) v_{i}(s+\tau) d k_{3 i}(s) \\
\leqslant & \int_{0}^{h} d \tau \int_{-h}^{0} \mathbb{E} \phi_{i}^{2}(\tau+s) z_{\mathfrak{i}}(t-\tau)\left[1-v_{i}(s+\tau)\right] d k_{3 i}(s) \\
& +\alpha_{03} \int_{0}^{t} \Gamma_{i}(\tau) z_{\mathfrak{i}}(t-\tau) d \tau
\end{aligned}
$$

Hence

$$
\mathbb{E} x_{i}^{2}(t) \leqslant C_{3 i}+\alpha_{03} \int_{0}^{t} \Gamma_{i}(\tau) z_{i}(t-\tau) d \tau
$$

and

$$
C_{3 i} \geqslant C_{2 i}+\int_{0}^{h} d \tau \int_{-h}^{0} \mathbb{E} \phi_{i}^{2}(\tau+s) z_{i}(t-\tau)\left[1-v_{i}(s+\tau)\right] d k_{3 i}(s) .
$$

Note that

$$
\int_{0}^{t} \Gamma_{i}(\tau) z_{i}(t-\tau) d \tau=\frac{1}{\gamma_{2 i}}\left(1-z_{i}(t)\right) \gamma_{2 i}(0)+\frac{1}{\gamma_{2 i}} \int_{0}^{t}\left(1-z_{i}(t-\tau)\right) d \Gamma_{i}(\tau) .
$$

Thus,

$$
\Gamma_{i}(t) \leqslant C_{3 i}+\alpha_{03} \int_{0}^{t} \Gamma_{i}(\tau) z_{i}(t-\tau) d \tau
$$

In view of (3.8), $\gamma_{2 i}>\alpha_{03}$, and Lemma 2.4, we have

$$
\Gamma_{i}(t) \leqslant \gamma_{2 i}+\frac{\gamma_{2 i} C_{3 i}}{\gamma_{2 i}-\alpha_{03}}:=C_{i}, i=1, \ldots, n,
$$

and

$$
\mathbb{E} x_{i}^{2}(t) \leqslant C_{i}, i=1, \ldots, n
$$

Thus,

$$
\mathbb{E} x^{2}(t) \leqslant \sum_{i=1}^{n} C_{i} .
$$

By Theorem 2.2, there exists a T-periodic solution of system (3.1).

From the proof of Theorem 3.1, we have the following corollary.

Corollary 3.2. Let the assumptions $\left(\mathrm{H}_{1}\right)$ and $\left(\mathrm{H}_{2}\right)^{*}$ hold. Furthermore, the following inequality holds:

$$
\gamma_{1 i}=\inf _{t \geqslant 0}\left[a_{i}(t)-\alpha_{01}^{1 / 2} \sum_{j=1}^{n}\left|d_{i j}(t)\right| / 2-\alpha_{01}^{1 / 2} / 2-\alpha_{02} / 2\right]>0,
$$

where $\alpha_{0 j}=\int_{-h}^{0} d k_{j i}(s), j=1,2,3$, and $k_{1 i}$ and $k_{2 i}$ are scalar non-decreasing functions bounded on $[-\mathrm{h}, 0]$. Then there exists a T-periodic solution of (3.1).

Remark 3.3. Compared with the methods in [17] and [9], our methods are easier than ones in the above two papers. In fact, in order to obtain existence of periodic solution for system (1.1), they obtained the following lemma. 
Lemma 3.4. A sufficient condition for the existence of an w-periodic Markov process with a given w-periodic transition function $\mathrm{p}\left(v, \mathrm{x}_{v}, \mathrm{t}, \mathrm{A}\right)$ is that for some $\mathrm{t}_{0}, \phi, \mathrm{x}_{\mathrm{t}}$ are uniformly stochastically continuous and

$$
\lim _{r \rightarrow \infty} \lim _{T \rightarrow \infty} \int_{t_{0}}^{t_{0}+T} p\left(t_{0}, \phi, \bar{U}_{r}\right) d t=0
$$

provided the transition function $\mathrm{p}\left(v, \mathrm{x}_{v}, \mathrm{t}, \mathrm{A}\right)$ satisfies the following not very restrictive assumption that

$$
\alpha(r)=\sup _{\phi \in \mathrm{U}_{\beta(r), 0<\mathrm{t}_{0}, \mathrm{t}-\mathrm{t}_{0}<\omega}} \mathrm{p}\left(\mathrm{t}_{0}, \phi, \overline{\mathrm{U}}_{\mathrm{r}}\right) \rightarrow 0 \text { as } \mathrm{r} \rightarrow \infty
$$

for some function $\beta(r)$ which tends to infinity as $r \rightarrow \infty$.

However, it is very difficult for obtaining the existence of an $\omega$-periodic Markov process by using Lemma 3.4. In the present paper, by using some inequalities lemmas (Lemma 2.3 and 2.4) and some mathematic analysis techniques we can easily obtain existence results of periodic solutions for neural networks system.

When the initial values in (3.1) are unbounded, we can formulate conditions for the unique existence of periodic solution of the form

$$
\left\{\begin{aligned}
\mathrm{d} x_{i}(t)= & {\left[-a_{i}(t) x_{i}(t)+\sum_{j=1}^{n} a_{i j}(t) f_{i}\left(x_{i}(t)\right)+\sum_{j=1}^{n} d_{i j}(t) g_{i}\left(x_{i}(t-\gamma)\right)\right] d t } \\
& +\sigma_{i}\left(t, x_{i}(t), x_{i}(t-\gamma)\right) d \xi_{i}(t), t \geqslant 0, \gamma \in(-\infty, 0] \\
x_{i}(\theta)= & \phi_{i}(\theta), \quad \theta \in(-\infty, 0], i=1,2, \ldots, n
\end{aligned}\right.
$$

We shall say that the T-periodic solution of (3.9) is unique if for any two T-periodic solution of this system $x(t)=\left(x_{1}(t), \ldots, x_{n}(t)\right)^{\top}$ and $y(t)=\left(y_{1}(t), \ldots, y_{n}(t)\right)^{\top}$ such that $\mathbb{E}|x(t)|^{2} \leqslant C<\infty$ and $\mathbb{E}|y(t)|^{2} \leqslant C<\infty$, and for all $t$ the following relations holds

$$
P_{i}\left(x_{i}(t)=y_{i}(t)\right)=1, \quad i=1, \ldots, n
$$

We need the following assumptions.

$\left(\mathrm{H}_{3}\right)$ For $i=1, \ldots, n$, assume $a_{\mathfrak{i}}(t)$ such that the solution $z_{\mathfrak{i}}(t, s)$ of homogeneous equation $x_{\mathfrak{i}}^{\prime}(t)=$ $-a_{i}(t) x_{i}(t)$ satisfies the following estimate

$$
\left|z_{i}(t, s)\right| \leqslant e^{-\lambda(t-s)}, \quad \lambda>0, \quad i=1, \ldots, n .
$$

$\left(\mathrm{H}_{4}\right)$ There exist $l_{i} \geqslant 0$ such that

$$
\left|f_{j}\left(x_{j}(t)\right)-f_{j}\left(y_{j}(t)\right)\right| \leqslant l_{i}, \quad i=1, \ldots, n .
$$

$\left(\mathrm{H}_{5}\right)$ For $i=1,2, \ldots, n$,

$$
\begin{aligned}
& \left|g_{i}\left(y_{i}(t-\tau)\right)-g_{i}\left(x_{i}(t-\tau)\right)\right|^{2} \leqslant \int_{-\infty}^{0}\left|\phi_{i}(s)-\psi_{i}(s)\right|^{2} d k_{1 i}(s)-l_{i}, \\
& \left|\sigma_{i}\left(t, y_{i}(t), y_{i}(t-\tau)\right)-\sigma_{i}\left(t, x_{i}(t), x_{i}(t-\tau)\right)\right|^{2} \leqslant \int_{-\infty}^{0}\left|\phi_{i}(s)-\psi_{i}(s)\right|^{2} d k_{2 i}(s),
\end{aligned}
$$

where $l_{i}$ is defined by $\left(\mathrm{H}_{3}\right)$, and $k_{1 i}$ and $k_{2 i}$ are scalar non-decreasing bounded on $(-\infty, 0]$ functions.

Theorem 3.5. Let the coefficients of (3.9) satisfy the conditions of Theorem 3.1 and assumptions $\left(\mathrm{H}_{3}\right)-\left(\mathrm{H}_{5}\right)$ hold. Then there exists unique T-periodic solution of (3.9). 
Proof. Assume that (3.9) has two T-periodic solution $x(t)$ and $y(t)$ satisfying initial condition $\phi(t)=$ $\left(\phi_{1}(t), \ldots, \phi_{n}(t)\right)^{\top}$ and $\psi(t)=\left(\psi_{1}(t), \ldots, \psi_{n}(t)\right)^{\top}$, respectively. Assume further that $x(t)$ and $y(t)$ satisfy $\mathbb{E}|x(t)|^{2} \leqslant C<\infty$ and $\mathbb{E}|y(t)|^{2} \leqslant C<\infty$. Let $u_{i}(t)=y_{i}(t)-x_{i}(t), i=1, \ldots, n$ satisfies the relation

$$
\begin{aligned}
u_{i}(t)= & z_{i}(t, s) u_{i}(s)+\int_{s}^{t} z_{i}(t, \tau)\left[\sum_{j=1}^{n} a_{i j}(\tau) f_{i}\left(y_{i}(\tau)\right)+\sum_{j=1}^{n} d_{i j}(\tau) g_{i}\left(y_{i}(\tau-\gamma)\right)\right. \\
& \left.-\sum_{j=1}^{n} a_{i j}(\tau) f_{i}\left(x_{i}(\tau)\right)-\sum_{j=1}^{n} d_{i j}(\tau) g_{i}\left(x_{i}(\tau-\gamma)\right)\right] d \tau \\
& +\int_{s}^{t} z_{i}(t, \tau)\left[\sigma_{i}\left(\tau, y_{i}(t), y_{i}(\tau-\gamma)\right)-\sigma_{i}\left(\tau, x_{i}(\tau), x_{i}(\tau-\gamma)\right)\right] d \xi_{i}(\tau) .
\end{aligned}
$$

Then for any positive constants $\varepsilon$ and $\varepsilon_{1}$, we have

$$
\begin{aligned}
\mathbb{E}\left|u_{i}(t)\right|^{2} \leqslant & \left(1+\varepsilon^{-1}\right)\left|z_{i}(t, s)\right|^{2} \mathbb{E}\left|u_{i}(s)\right|^{2}+(1+\varepsilon)\left[\left(1+\varepsilon_{1}\right) \int_{s}^{t}\left|z_{i}\left(t, \tau_{1}\right)\right| d \tau_{1} \int_{s}^{t}\left|z_{i}(t, \tau)\right|\right. \\
& \times \mathbb{E} \mid \sum_{j=1}^{n} a_{i j}(\tau) f_{i}\left(y_{i}(\tau)\right)+\sum_{j=1}^{n} d_{i j}(\tau) g_{i}\left(y_{i}(\tau-\gamma)\right) \\
& -\sum_{j=1}^{n} a_{i j}(\tau) f_{i}\left(x_{i}(\tau)\right)-\left.\sum_{j=1}^{n} d_{i j}(\tau) g_{i}\left(x_{i}(\tau-\gamma)\right)\right|^{2} d \tau \\
& \left.+\left(1+\varepsilon_{1}^{-1}\right) \int_{s}^{t}\left|z_{i}(t, \tau)\right|^{2} \mathbb{E}\left|\sigma_{i}\left(\tau, y_{i}(t), y_{i}(\tau-\gamma)\right)-\sigma_{i}\left(\tau, x_{i}(\tau), x_{i}(\tau-\gamma)\right)\right|^{2} d \tau\right] .
\end{aligned}
$$

Let $s \rightarrow-\infty$ in the above inequality, by $\left(\mathrm{H}_{3}\right)-\left(\mathrm{H}_{5}\right)$, we get

$$
\mathbb{E}\left|u_{i}(t)\right|^{2} \leqslant(1+\varepsilon)\left[\left(1+\varepsilon_{1}\right) \lambda^{-2} \alpha_{01}+\left(1+\varepsilon_{1}^{-1}\right)(2 \lambda)^{-1} \alpha_{02}\right] f_{i}(t),
$$

where $f_{i}(t)=\sup _{s \leqslant t} \mathbb{E}\left|u_{i}(s)\right|^{2}$. Obviously, when $\varepsilon_{1}=\left(\lambda \alpha_{02}\right)^{1 / 2}\left(2 \alpha_{01}\right)^{11 / 2}$, we have the minimum of the righthand side of (3.10). Thus,

$$
f_{i}(t) \leqslant(1+\varepsilon)\left[\lambda^{-1} \alpha_{01}^{1 / 2}+(2 \lambda)^{-1} \alpha_{02}^{1 / 2}\right]^{2} f(t), \quad i=1, \ldots, n .
$$

Let

$$
v=\lambda^{-1} \alpha_{01}^{1 / 2}+(2 \lambda)^{-1} \alpha_{02}^{1 / 2}<1
$$

Then we choose $\varepsilon>0$ such that $(1+\varepsilon) v<1$. By (3.11) we have $P\left(x_{\mathfrak{i}}(t)=y_{\mathfrak{i}}(t)\right)=1$ for all $t$ and $i=1,2, \ldots, n$. Hence $P(x(t)=y(t))=1$. The proof is completed.

Remark 3.6. In [7], the authors studied the uniqueness of periodic solution for equations containing linear terms $A(t) x(t)$ of the form

$$
\left\{\begin{array}{l}
\mathrm{d} x(t)=\left[A(t) x(t)+a\left(t, x_{t}\right)\right] d t+b\left(t, x_{t}\right) d \xi(t), t \geqslant 0, \\
x_{t}(\theta)=x(t+\theta), \theta \leqslant 0, x(t) \in \mathbb{R}^{n}, \xi(t) \in \in \mathbb{R}^{l},
\end{array}\right.
$$

where $A(t)$ is a matrix with continuous T-periodic entries such that the fundamental matrix $Z(t, s)$ of the homogeneous equation $x^{\prime}(t)=A(t) x(t)$ satisfies for some constant $\lambda>0$ the estimate

$$
Z(t, s) \leqslant e^{-\lambda(t-s)} .
$$

Under the conditions

$$
|a(t, \phi)-a(t, \psi)|^{2} \leqslant \int_{-\infty}^{0}|\phi(s)-\psi(s)|^{2} d k_{1}(s), \quad|b(t, \phi)-b(t, \psi)|^{2} \leqslant \int_{-\infty}^{0}|\phi(s)-\psi(s)|^{2} d k_{2}(s),
$$

then (3.12) has unique T-periodic solution. The proof of Theorem 3.5 is similar to the proof in [7]. It is worth pointing out that assumptions $\left(\mathrm{H}_{4}\right)$ and $\left(\mathrm{H}_{5}\right)$ are different from the conditions in [7] in order to obtain unique periodic solution. 


\section{Numerical example}

In order to verify the feasibility of our results, we examine the existence of periodic solutions for the following stochastic nonlinear system:

$$
\left\{\begin{aligned}
d x_{1}(t)= & -a_{1}(t)+\sum_{j=1}^{2} a_{1 j}(t) f\left(x_{1}(t)\right)+\sum_{j=1}^{n} d_{1 j}(t) g_{1}\left(x_{1}(t-\gamma)\right) d t \\
& +\sigma_{1}\left(t, x_{1}(t), x_{1}(t-\gamma)\right) d \xi_{1}(t), \\
d x_{2}(t)= & -a_{2}(t)+\sum_{j=1}^{2} a_{2 j}(t) f\left(x_{2}(t)\right)+\sum_{j=1}^{n} d_{2 j}(t) g_{2}\left(x_{2}(t-\gamma)\right) d t \\
& +\sigma_{2}\left(t, x_{2}(t), x_{2}(t-\gamma)\right) d \xi_{2}(t), \quad t \geqslant 0, \gamma \in[-1,0], \\
x_{i}(\theta)= & \phi_{i}(\theta), \quad \theta \in[-1,0], \quad i=1,2,
\end{aligned}\right.
$$

where

$$
\begin{aligned}
a_{\mathfrak{i}}(t) & =20+\sin t, & a_{i j}(t) & =d_{i j}(t)=2+\sin t, \\
f_{i}\left(x_{i}(t)\right) & =-x_{i}^{3}(t)-x_{i}(t), & g_{i}\left(x_{i}(t-\gamma)\right) & =\sin \left(x_{i}(t-\gamma)\right), \quad \sigma_{i}\left(t, x_{i}(t), x_{i}(t-\gamma)\right)=\frac{1}{2} \cos t .
\end{aligned}
$$

Obviously, $\sum_{j=1}^{2} a_{1 j}(t)=\sum_{j=1}^{2} a_{2 j}(t)=4+2 \sin t>0$ and $x_{i} f_{i}\left(x_{i}\right)=-x_{i}^{4}-x_{i}^{2} \leqslant 0$, hence assumption $\left(\mathrm{H}_{2}\right)$ holds. Choosing $k_{i}(s)=s, i=1,2$, we have

$$
\alpha_{01}=\alpha_{02}=\int_{-1}^{0} d k_{1}(s)=1
$$

and

$$
\gamma_{1}=\inf _{t \geqslant 0}\left[a_{1}(t)-\alpha_{01}^{1 / 2} \sum_{j=1}^{n}\left|d_{i j}(t)\right| / 2-\alpha_{01} / 2-\alpha_{02} / 2\right]=18-\frac{\sqrt{6}}{2}>0 .
$$

Then we can choose proper $\mathrm{q}_{1}, \mathrm{q}_{2}$ and initial function $\phi$ in order to other conditions of Theorem 3.1 satisfy. It follows from Theorem 3.1 that system (4.1) exists a periodic solution.

The numerical simulations of system (4.1) are shown in Fig. 1. Fig. 1 shows the state trajectories of periodic solution $x(t)=\left(x_{1}(t), x_{2}(t)\right)^{\top}$ for system (4.1).

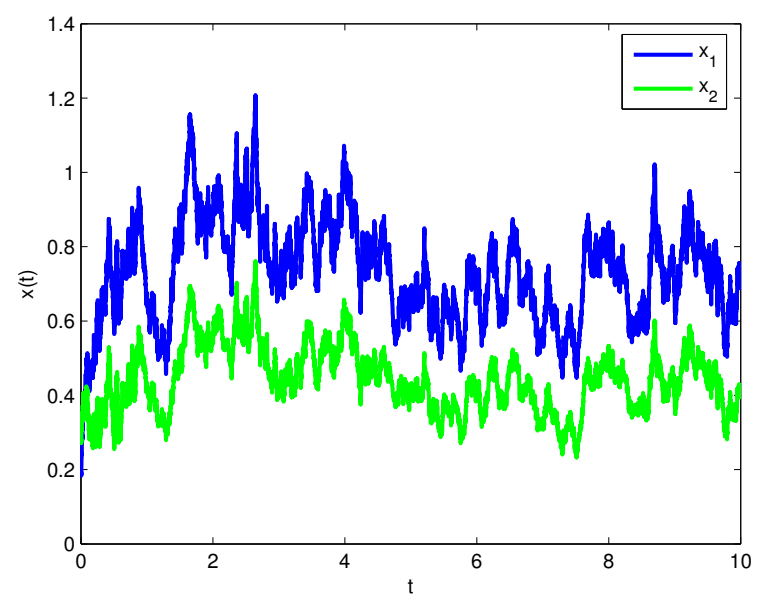

Figure 1: The States' evolution of the system (4.1). 


\section{Conclusion}

In this paper, novel results for existence and uniqueness of periodic solutions for a class of stochastic nonlinear system are obtained. As Kolmanovskii and Myshkis [7] pointed out that the proof of existence theorems of periodic solutions of stochastic equations with delays is similar to the proof of existence theorems of stationary solutions, the modifications arising are due to the fact that the distribution of probabilities of periodic solutions must be invariant with respect to shifts of argument by quantities multiple to the period while for the stationary solutions it is invariant with respect to arbitrary shifts of the argument. That is why in this paper we shall give some coefficient conditions for the existence of periodic solutions. At last, we provide a numerical example to illustrate the effectiveness of the theoretical results.

\section{Acknowledgment}

The authors would like to thank the editors and the anonymous referees for their valuable comments and suggestions to improve the presentation of this paper. The first author is supported by author Natural Science Foundation of Jiangsu High Education Institutions of China (Grant No. 17KJB110001). The second author is supported by the National Science Foundation (DMS \# 1737861 ).

\section{References}

[1] R. Z. Has'minskii, On the dissipativity of random processes defined by differential equations, Problems of Information Transmission, 1 (1965), 88-104. 1

[2] R. Z. Has'minskii, Stochastic Stability of Differential Equations, Sijthoff and Noordhoff, Springer Science \& Business Media, NetherIands, (1980). 1

[3] K. Itô, On Stochastic Differential Equations, Mem. Amer. Math. Soc., American Mathematical Society, Rhode Island, (1951). 1

[4] K. Itô, M. Nisio, On stationary solutions of a stochastic differential equation, J. Math. Kyoto Univ., 4 (1964), 1-75. 1

[5] D. Jiang, N. Shi, A note on nonautonomous logistic equation with random perturbation, J. Math. Anal. Appl., 303 (2005), 164-172. 1

[6] D. Jiang, N. Shi, X. Li, Global stability and stochastic permanence of a non-autonomous logistic equation with random perturbation, J. Math. Anal. Appl., 340 (2008), 588-597. 1

[7] V. Kolmanovskii, A. Myshkis, Introduction to the Theory and Application of Functional Differential Equations, Kluwer Academic Publishers, Dordrecht, (1999). 2, 2.1, 2.2, 2.3, 2.4, 3.6, 3.6, 5

[8] C. Li, L. Chen, K. Aihara, Impulsive control of stochastic systems with applications in chaos control, chaos synchronization, and neural networks, Chaos, 2008 (2008), 11 pages. 1

[9] D. Li, D. Xu, Periodic solutions of stochastic delay differential equations and applications to Logistic equation and neural networks, J. Korean Math. Soc., 50 (2013), 1165-1181. 1, 3.3

[10] J. Liang, Z. Wang, Y. Liu, X. Liu, Robust synchronization of an array of coupled stochastic discrete-time delayed neural networks, IEEE Trans. Neural Netw., 19 (2008), 1910-1921. 1

[11] K. Lu, B. Schmalfuss, Invariant manifolds for stochastic wave equations, J. Differential Equations, 236 (2007), 460-492. 1

[12] X. R. Mao, Exponential Stabiity of Stochastic Differential Equations, Marcel Dekker, New York, (1994).

[13] B. Oksendal, Stochastic Differential Equations: An Introduction with Applications (Sixth Edition), Springer-Verlag, Berlin, (2003). 1

[14] Y. Tang, W. K. Wong, Distributed synchronization of coupled neural networks via randomly occurring control, IEEE Trans. Neural Netw. Learn. Syst., 24 (2013), 435-447. 1

[15] B. Tojtovska, S. Janković, On some stability problems of impulsive stochastic Cohen-Grossberg neural networks with mixed time delays, Appl. Math. Comput., 239 (2014), 211-226. 1

[16] X. Wu, Y. Tang, W. Zhang, Stability analysis of switched stochastic neural networks with time-varying delays, Neural Network, 52 (2014), 39-49. 1

[17] D. Xu, Y. Huang, Z. Yang, Existence theorems for periodic Markov process and stochastic functional differential equations, Discrete Contin. Dyn. Syst., 24 (2009), 1005-1023. 1, 1, 3.3

[18] Z. Zhang, J. Cao, Do. Zhou, Novel LMI-Based condition on global asymptotic stability for a class of Cohen-Grossberg BAM networks with extended activation functions, IEEE Transcation on Neural Network and learning system, 25 (2014), 1161-1172. 1

[19] B. G. Zhang, K. Gopalsamy, On the periodic solution of n-dimensional stochastic population models, Stochastic Anal. Appl., 18 (2000), 323-331. 1, 1 
[20] Z. Zhang, K. Liu, Existence and global exponential stability of a periodic solution to interval general bidirectional associative memory (BAM) neural networks with multiple delays on time scales, Neural Network, 24 (2011), 427-439. 1

[21] W. Zhang, Y. Tang, W. Wong, Q. Miao, Stochastic stability of delayed neural networks with Local impulsive effects, IEEE Trans. Neural Netw. Learn. Syst., 26 (2015), 2336-2345. 1

[22] Q. Zhu, J. Cao, Exponential stability of stochastic neural networks with both Markovian jump parameters and mixed time delays, IEEE Trans. Syst., Man, Cybern. B, Cybern., 41 (2011), 341-353. 1

[23] Q. Zhu, J. Cao, Stability of Markovian jump neural networks with impulse control and time varying delays, Nonlinear Anal. Real World Appl., 13 (2012), 2259-2270. 1

[24] H. Zhou, Z. Zhou, Z. Qiao, Mean-square almost periodic solution for impulsive stochastic Nicholson's blowflies model with delays, Appl. Math. Comput., 219 (2013), 5943-5948. 1 